\title{
Descriptive study of the youth male judoka aged 10-14 years by competitive age group and maturity status
}

\author{
Bruno GIUDICELLI* \\ Universidade Federal de Alagoas (Brazil)
}

\author{
5th IMACSSS World Scientific Congress Abstracts, Rio Maior (Portugal), October 6-8 \\ Section: Biological, kinesiological and physiological aspects of MA\&CS \\ Type: Oral communication
}

\section{Introduction}

Adolescences, due to the growth and maturation, experience increments in body size, in body composition, in functional capacity and in motor dexterity (Malina, Bouchard, \& Bar-Or, 2004), and these changes have a major impact on the physical performances. It is common during this period we find young athletes of the same age and sports category at different levels of growth and maturation, which may result in inequities related to body size and functional capabilities (Malina, et al., 2010). In combat sports, specifically in judo, where performance can be determine by physical, technical, tactical and psychological abilities (Detanico, Dal Pupo, Franchini, \& Giovana dos Santos, 2012), with extensive physical contact, it becomes even more pertinent to assess how the processes of growth and maturation affect the performance of young athletes in order to instrument the physical education teachers and coaches with relevant pedagogical and methodological training information, in order to protect these athletes, to gain in physical and sports performance and to contribute to the practice for the long term.

\section{Methodology}

The sample included 76 young male judokas aged 10.37-14.7 years from four competitive age groups, U11 $(n=9), \mathrm{U} 12(n=23), \mathrm{U} 13(n=22)$ and U15 $(n=22)$. Weight, height, sitting height, arm span, hand length, foot length; elbow, knee, biacromial and bicristal breadth; arm flexed and calf circumferences; four skinfolds (triceps, subscapular, suprailiac and medial calf) and seven functional capacities (aerobic, anaerobic, agility and abdominal, upper body, lower body and handgrip muscle strength) were measure. Maturity status was assessed through somatic maturity using percentage of the predicted adult height (PPAH). Each judoka was classified as late, on time or early maturing by PPAH z-scores. ANOVA was used to compare characteristics of judokas across competitive age groups. A level of significance of $p<0.05$ was settled.

\section{Results}

Older athletes are significantly taller and heavier and have proportionately longer arms and legs. The hand and foot lengths are significantly higher. The arm flexed and calf circumference are higher $(F=7.74$ and 8.55 , respectively, $p<0.01)$, indicating a significantly greater muscle mass in the upper and lower limbs, since the groups did not differ with regard to skinfolds and the sum of skinfolds. The somatotypes components did not vary significantly. The maturational status between the competitive age groups vary significantly, with a prevalence of late maturing athletes in the U11 and U12 groups and a dominance of on time maturing athletes in the U13 and U15 groups. There is a predominance of on time maturing athletes in the total sample (53\%), followed by late maturing athletes $(39.5 \%)$ and early maturing athletes (5.3\%) 
All functional capacities improve with increasing age, with the exception only of aerobic capacity, abdominal and lower body strength that present moments of decrease in performance. However, among them only the aerobic capacity varies significantly $(F=4.7, p \leq 0.05, r=0.40)$. Beyond it, significantly differences can be found in anaerobic capacity, upper body strength and hand grip strength. There are no significant differences in agility, abdominal and lower body strength.

\section{Discussion and conclusion}

Sánchez-Muñoz, Zabala and Williams (2012) emphasize the importance of monitoring the maturation of young judokas because, given the nature of judo is try to remove your opponent from a position of balance, biological maturation possible exert impact on performance and chances of success.

However, Krstulović, Sekulić and Sertić (2005) relativize the effect of the maturity status in judo, since most studies that have been focused on comparisons of young judokas of different maturity status within a chronological age showed that the differences in the functional capacities are reduced when body size is statistically controlled. According to the authors, the categorization of judo athletes in weight categories would ensure the control of the influence of body size, minimizing the maturational effect.

Athletes of the two oldest competitive age groups are significantly more mature, taller, heavier, have longer upper and lower body limbs and higher muscle mass than the athletes of the two youngest competitive age groups. They also have higher aerobic and anaerobic capacities, higher upper body and handgrip strength. The predominant differentiation factor seems to be the adolescent growth spurt, which usually occurs around the age of 12, exactly the age of cut between the two pairs of competitive age groups with significantly different results.

The maturational status difference within each age group and its influence on the growth, body development and physical performance will be the aims of next studies on this sample. On the occasion will be verified the influence of categorization by weight in the attenuation of maturing effects on functional capacity.

\section{References}

Detanico, D., Dal Pupo, J., Franchini, E., \& Giovana dos Santos, S. (2012). Relationship of aerobic and neuromuscular indexes with specific actions in judo. Science \& Sports, 27(1), 16-22.

Krstulović, S., Sekulić, D., \& Sertić, H. (2005). Anthropological determinants of success in young judoists. Collegium Antropologicum, 29(2), 697-703.

Malina, R. M., Bouchard, C., \& Bar-Or, O. (2004). Growth, Maturation, and Physical Activity (2nd ed.). Champaign, Illinois: Human Kinetics.

Malina, R. M., Peña Reyes, M. E., Figueiredo, A. J., Coelho E Silva, M. J., Horta, L., Miller, R., ... Morate, F. (2010). Skeletal age in youth soccer players: implication for age verification. Clinical Journal of Sport Medicine: Official Journal of the Canadian Academy of Sport Medicine, 20(6), 469-74.

Sánchez-Muñoz, C., Zabala, M., \& Williams, K. (2012). Anthropometric Variables and Its Usage to Characterise Elite Youth Athletes. In V. R. Preedy (Ed.) Handbook of Anthropometry: Physical Measures of Human Form in Health and Disease (pp. 1865-1888). New York, NY: Springer.

Key words: Young athletes; somatic maturation; anthropometry; functional capacities; judo; combat sports. 